\title{
The use of saliva as a practical and feasible alternative to urine in large-scale screening for congenital cytomegalovirus infection increases inclusion and detection rates
}

\author{
Emanuelle Santos de Carvalho Cardoso ${ }^{[1]}$, Bruna Laís Santos de Jesus ${ }^{[1]}$, \\ Luciano Gama da Silva Gomes ${ }^{[1]}$, Sandra Mara Bispo Sousa ${ }^{[2],}$ \\ Sandra Rocha Gadelha ${ }^{[1]}$ and Lauro Juliano Marin ${ }^{[1]}$
}

[1]. Departamento de Ciências da Saúde, Universidade Estadual de Santa Cruz, Ilhéus, Bahia, Brasil. [2]. Departamento de Ciências Naturais, Universidade Estadual do Sudoeste da Bahia, Vitória da Conquista, Bahia, Brasil.

\begin{abstract}
Introduction: Although urine is considered the gold-standard material for the detection of congenital cytomegalovirus (CMV) infection, it can be difficult to obtain in newborns. The aim of this study was to compare the efficiency of detection of congenital CMV infection in saliva and urine samples. Methods: One thousand newborns were included in the study. Congenital cytomegalovirus deoxyribonucleic acid (DNA) was detected by polymerase chain reaction (PCR). Results: Saliva samples were obtained from all the newborns, whereas urine collection was successful in only 333 cases. There was no statistically significant difference between the use of saliva alone or saliva and urine collected simultaneously for the detection of CMV infection. Conclusions: Saliva samples can be used in large-scale neonatal screening for CMV infection.
\end{abstract}

Keywords: Congenital cytomegalovirus. Congenital infection. Neonatal screening.

The late onset of symptoms such as hearing deficiency and psychomotor development delay in congenital infection with cytomegalovirus (CMV) can result in progressive and irreversible sequelae. Early diagnosis and follow-up of affected children are therefore essential for the detection and prevention of future problems ${ }^{(1)(2)}$. In newborns (NBs), the diagnosis can be made based on polymerase chain reaction (PCR) analysis of various clinical samples, among which urine is considered the gold standard. However, difficulties in urine collection documented in some studies have led to a suggestion that saliva analysis may represent an easier, more practical, and less expensive approach ${ }^{(3)}{ }^{(4)}$. The aim of this large-scale neonatal screening study was to compare the efficiency of saliva and urine samples analysis in detecting congenital infection with CMV.

This study included 1,000 NBs delivered at a public maternity hospital in Ilhéus, State of Bahia, from February to September 2012. Infants of any gestational age and clinical characteristics were included provided that a sample of urine or/and saliva was collected before the age of three weeks.

Corresponding author: Dr. Lauro Juliano Marin. Departamento de Ciências da Saúde/UESC. Rodovia Jorge Amado, km 16, Ilhéus, Bahia, Brasil.

Phone: 5573 8804-7335; 5573 3680-5325; Fax: 5573 3680-5116

e-mail: lajumarin@hotmail.com

Received 14 September 2014

Accepted 29 October 2014
If viral deoxyribonucleic acid (DNA) was detected in at least two different samples, the infant was considered as having congenital CMV infection.

Saliva was collected with sterile swabs (Labor Swab, China) placed delicately on the inner surface of the cheek of the NB for $1 \mathrm{~min}$. The swabs were then transferred to sterile plastic tubes containing $700 \mu$ l of a transport medium (MEM Earle, Cultilab, USA). Urine samples were collected using hypoallergenic collecting bags (Mark Med, Brazil) and transferred to sterile plastic bottles. Samples of both types were kept at $4^{\circ} \mathrm{C}$ and subjected to PCR analysis within $24 \mathrm{~h}$.

Viral genomes were detected by nested PCR using MIE4MIE5 ${ }^{(5)}$ external primers and IE1-IE2 ${ }^{(6)}$ internal primers without prior extraction of DNA. Samples were incubated at $94^{\circ} \mathrm{C}$ for $5 \mathrm{~min}$ before the analysis.

Congenital CMV infection was detected by PCR analysis of urine or/and saliva in $13(1.3 \%) \mathrm{NB}$, whereas the remaining 987 subjects were considered uninfected. The infected infants were subjected to hearing and sight tests as well as a complete physical examination. Twelve $(92.3 \%)$ of them were asymptomatic and $1(7.7 \%)$ had microcephaly.

It was not possible to obtain urine samples from $667 / 1,000$ (66.7\%) NBs because of difficulties such as meconium contamination, delay of diuresis, leakage of urine collector bags, and bag-induced skin irritation. In contrast, saliva samples were 
collected in $100 \%$ of the NBs, which reflects the easiness with which they could be obtained.

Both urine and saliva were collected and analyzed in 333 cases, and congenital infection was detected in 5 of them (prevalence, 1.5\%; 95\% confidence interval (CI), 0.6-3.4). In one case, infection was detected in the urine but not in the saliva. The strength of agreement between urine and saliva analysis, as assessed by a Kappa statistic, was very high (Kappa $=0,887$; $\mathrm{p}<0.001)$. Congenital infections were detected in 8 out of the $667 \mathrm{NBs}$ in whom only saliva was collected (prevalence, $1.2 \%$; CI, 0.6-2.3). There was no statistically significant difference between the use of saliva only $(8 / 667 ; 1.2 \% ; 95 \% \mathrm{CI}, 0.5-3.0)$ or saliva and urine collected simultaneously $(5 / 333 ; 1.5 \% ; 95 \%$ CI 0.6-3.4) $(\mathrm{p}=0.70)$. Considering urine as the gold standard, the sensitivity and specificity of saliva analysis were $80 \%$ and $100 \%$, respectively, and the positive and negative predictive values were $100 \%$ and $99.7 \%$, respectively.

The difficulty in obtaining urine in a large-scale screening study can negatively affect the results because of the exclusion of many NBs from whom a sample cannot be obtained ${ }^{(7)(8)}$. Thus, we were unable to collect urine from two-thirds of the participating babies, which, had not saliva samples been collected, would have resulted in eight cases of CMV infection left undetected. Therefore, saliva samples procurement as an alternative to urine can dramatically (up to $100 \%$ ) improve the inclusion index in neonatal screening for CMV. It was previously demonstrated that analysis of properly collected saliva samples can yield a sensitivity of $>97 \%$ and a specificity of $99.9 \%$ for the detection of congenital CMV infection with molecular techniques ${ }^{(3)}$. Our results are in line with these published values (specificity of $100 \%$ for both samples).

No false-positive PCR results were obtained for saliva samples. In particular, all the positive results were confirmed with viral DNA amplification from the simultaneously obtained urine sample as well as new urine and saliva samples collected from the same child later. However, DNA amplification from the urine sample revealed one case of false-negative result of saliva analysis. This could probably have occurred because of a problem during saliva collection, such as failure to wait for the appropriate amount of time for the swab to become properly moistened, sample collection immediately after breastfeeding, or contamination by a substance that inhibited the PCR. It has to be noted that a number of measures were taken to minimize the possibility of inhibition, such as heating, dilution, and centrifugation of the samples prior to analysis. Nevertheless, we cannot completely rule out the possibility of other false-negative results in the cases where urine samples were not collected. Previous studies also showed instances of false-negative detection of congenital CMV infection by PCR analysis of saliva and urine samples, which could have been caused by improper collection of the material or by the presence of metabolites, drugs, or other PCR-inhibiting substances $^{(3)(7)}$.

Although few similar studies have been conducted in Bahia, the incidence of congenital CMV infection of $1.5 \%$ determined in the present study is in agreement with the results of studies performed in other regions of Brazil and other countries. Thus, the evaluation of 4,400 NBs from São Paulo revealed the prevalence of CMV DNA detection by PCR of $1.1 \%$ in urine and saliva samples ${ }^{7}$. Furthermore, Paixão et al. obtained a congenital CMV infection prevalence of 1.05\% (CI 95\%: 0,748-1,446) using dried blood spot testing (Guthrie cards) in 3,600 Portuguese NBs performed over a period of 14 months $^{(8)}$.

In conclusion, saliva seems to represent a viable alternative to urine, the gold-standard material for congenital CMV infection screening, owing to the ease of collection combined with comparable sensitivity and specificity. Moreover, the use of saliva may result in the inclusion of up to $100 \%$ of the participating children, allowing the detection of a higher number of congenital infection cases.

\section{Ethical considerations}

The study was approved by the Committee of ethics in research with Human Beings of the Universidade Estadual de Santa Cruz (UESC), Protocol 209/08. All mothers or guardians of the NBs provided written informed consent.

\section{CONFLICT OF INTEREST}

The authors declare that there is no conflict of interest.

\section{REFERENCES}

1. Van Rijckevorsel GG, Bovée LP, Damen M, Sonder GJ, Schim van der Loeff MF, van den Hoek A. Increased seroprevalence of IgGclass antibodies against cytomegalovirus, parvovirus B19, and varicella-zoster virus in women working in child day care. BMC Public Health 2012; 12:475-482.

2. Boppana SB, Ross SA, Novak Z, Shimamura M, Tolan Jr RW, Palmer AL, et al. Dried blood spot real-time polymerase chain reaction assays to screen newborns for congenital cytomegalovirus infection. JAMA 2010; 303:1375-1382.

3. Boppana SB, Ross SA, Shimamura M, Palmer AL, Ahmed A, Michaels MG, et al. Saliva polymerase-chain-reaction assay for cytomegalovirus screening in newborns. N Engl J Med 2011; 364:2111-2118.

4. Yamamoto AY, Mussi-Punhata MM, Marin LJ, Brito RM, Oliveira PFC, Coelho TB. Is saliva as reliable as urine for detection of cytomegalovirus DNA for neonatal screening of congenital CMV infection? J Clin Virol 2006; 36:228-230.

5. Demmler GJ, Buffone GJ, Schimbor CM, May RA. Detection of cytomegalovirus in urine from newborns by using polymerase chain reaction DNA amplification. J Infect Dis 1988; 158:1177-1184.

6. Nogueira E, Arruda VR, Bizzacchi JM, Costa FF, Ozelo MC, Rossi CL, et al. Possible association between cytomegalovirus infection and gastrointestinal bleeding in hemophiliac patients. Acta Haematol 2000; 103:73-77.

7. Almeida S, Gouveia P, Jorge A, Mendes A, Duarte C, Faria N, et al. Infecção congênita por citomegalovirus. Prevalência numa população da Beira Interior. Acta Pediatr Port 2010; 41:162-165.

8. Paixão P, Almeida S, Gouveia P, Vilarinho L, Vaz Osório R. Prevalence of human cytomegalovirus congenital infection in Portugues newborns. Euro Surveill 2009; 14:13-15. 